\title{
Reproducción y producción, aproximación a los cuidados desde unas perspectiva y focalización artísticas
}

\section{Reproduction and production, approach to care from an artistic perspective and focus}

\author{
TIPO DE TRABAJO: Comunicación virtual \\ PALABRAS CLAVE \\ Arte-Vida; Maternidad; Producción-Reproducción; Reproducción Social; Arte-Cuidados.
}

KEY WORDS

Art-Life; Mothehood; Production-Reproduction; Social Reproduction; Art-Life Care.

RESUMEN

El tema fundamental de la condición de la maternidad en las sociedades y teorías contemporáneas es el de los cuidados. El asunto aquí se investiga desde una perspectiva y con una focalización artísticas. La comunicación consta de seis apartados en el que se hace un resumen del proceso de una investigación en curso en este momento. Empezando por una introducción a nivel general y a nivel particular del tema, se detallan referentes artísticos en torno al tema de la maternidad desde los años sesenta hasta la actualidad, se expone el modo de obtención de datos en el estudio de casos y algunos resultados del mismo y se reflexiona sobre un modo de trabajo artístico y su modo de difusión, con el que dar visibilidad a nuevas narrativas que den lugar a nuevos conceptos y discursos acerca del tema en cuestión.

\section{ABSTRACT}

The fundamental issue of the condition of motherhood in contemporary societies and theories is child care. The subject here is investigated from a perspective and with an artistic focus. The article consists of six sections that summarize the process of an ongoing investigation. Beginning with an introduction at a general level and at a particular level of the issue, art references are given to the subject of motherhood from the 1960s to the present, it exposes the way of obtaining data in the case study and some results of it and reflects on a way of artistic work and its mode of diffusion, with which to give visibility to new narratives that give rise to new concepts and discourses about the subject in question.

\section{INTRODUCCIÓN}

"sabemos mucho más acerca del aire que respiramos o de los mares que atravesamos que acerca de la naturaleza y del significado de maternidad"1

Desde los años 60 y 70 el feminismo y la teoría de género junto con los avances tecnológicos en temas de anticoncepción y reproducción humana, entre otros factores, están originando una reorganización social en la que está cambiando el modelo de organización familiar y por lo tanto la sociedad se está reestructurando desde su núcleo.

${ }^{1}$ Esta misma frase la utiliza Irate Fernández Pujana en el comienzo de Feminismo y maternidad cuna relación incómoda? 
La incorporación de la mujer al mercado laboral, la política, la comunidad científica, la academia y las artes entre otros ámbitos, están creando una nueva forma de comprender la vida.

El tema de los cuidados es el tema fundamental de la condición de la maternidad en las sociedades y en las teorías contemporáneas, los cuidados en este momento siguen recayendo en su mayor parte en las madres (Legarretalza 2009), que derivan funciones por falta de tiempo a su red familiar, a instituciones públicas o privadas o a empleadas para el servicio doméstico, importante es mencionar que en el sistema económico actual no se contemplan como productivas las labores de reproducción y cuidados, disfunción del sistema que provoca una situación de discriminación económica estructural ${ }^{2}$ puesto que no generan ningún tipo de rentas por lo tanto chocan de lleno con el sistema capitalista lo que está generando una bajada drástica de la natalidad.

Dado que los tipos de familia están cambiando a gran velocidad, es necesario unos estudios y reflexiones profundas sobre el tema de la maternidad y los cuidados puesto que de ellos depende, en gran medida y exponencialmente, el modelo de sociedad que queremos perfilar.

Los cuestionamientos que genera el tema eclosionan por necesidad, temas como el de la gestación subrogada y custodias compartidas impuestas, así como el envejecimiento de la población, lo ponen en el punto de mira. Parece imprescindible, como nuclear que es, que se analice y se trabaje para engranarlo al sistema con el fin de conseguir el desarrollo de un nuevo organismo social ${ }^{3}$.

Desde mi posición en la sociedad como una mujer trabajadora que ha tenido la posibilidad de acceder a unos estudios universitarios una vez ha sido madre, yuxtaponiendo ambas actividades, quedando junto a mi hija como una unidad familiar monoparental a la deriva $^{4}$ en medio de la crisis económica, pretendo con mi investigación, buscar una dimensión común ${ }^{5}$ de la experiencia vivida como madre y artista mediante una serie de estudios de casos y mi propio relato vital como un sujeto al que se le rompen todas las dinámicas aprendidas en un momento determinado ${ }^{6}$, ejercitando mis capacidades creativas desde mi reproducción y producción artística.

\section{PROYECTOS ARTÍSTICOS Y BÚSQUEDA DE UN LENGUAJE PLÁSTICO Y VISUAL ANTERIORES A INVESTIGACIÓN DE PRODUCCIÓN Y REPRODUCCIÓN, MI CASO}

La gran ansiedad que me generaba el no poder trabajar y producir más obra puesto que debía dedicar mi tiempo al cuidado de mi hija, hacía que mi contestación a la pregunta de ¿Por qué no han existido grandes artistas mujeres? Que formulaba Linda Nochlin allá por los setenta era, en un momento determinado, la misma que daría Rosa Olivares

“[...] estaban pariendo, estaban haciendo la comida, estaban fregando. Estaban facilitando el triunfo de sus parejas, en una demostración todavía no suficientemente estudiada de cómo el Síndrome de Estocolmo afecta especialmente a las mujeres, tradicionalmente esclavas del entorno afectivo y familiar." (Olivares, 2008:4) ${ }^{7}$

Sin ser apenas consciente de ello en su momento, gran parte de los proyectos artísticos que desarrollé en la Facultad de Bellas Artes estaba relacionado con mi hija o la maternidad y cuidados.

\footnotetext{
2 "Con más consistencia se puede afirmar que la fuerza motriz de la economía mundial ha sido la capacidad del capitalismo internacional de apropiarse de las masas trabajadoras globales de campesinos expropiados y de amas de casa, es decir, de la inmensa cantidad de trabajo no contractual, incrementando así de manera exponencial los porcentajes de extracción de plusvalía" (Federici 2013).

3 “I am searching for field character", declaración de Beuys de 1973 (citado en Tisdal 1974, 48) (Carrascosa 2016, Nota número 4).

${ }^{4}$ El término de precaria lo he tomado de $A$ la deriva: por los circuitos de la precariedad femenina.

5 "En este contexto, rescatar los cuidados es un modo de visibilizar la interdependencia que se encuentra en el corazón de toda vida. Lo que expone son los lazos robados, quebrados, de nuestro mundo hecho añicos. Aún en el terreno de la dispersión, las diferencias, los relatos fragmentados, no hay vida posible sin esa dimensión común de la experiencia. Aunque sea la experiencia común de un malestar. Y es en este sentido en el que lo común ya no es solo un reto a construir entre el oleaje de diferencias en movimiento perpetuo, sino algo que rescatar de entre lo que hay. Ya no solo un lugar al que llegar, sino también un lugar del que partir". Gil, Silvia. 2012.

6 "Pero la desestabilización de algún de estos elementos hace que se tambalee el ideal de independencia: dejamos de ser jóvenes, no gozamos de tal estatus social, nos sobreviene una enfermedad crónica o pasajera, decidimos tener hijos o cuidamos de otras personas. En ese caso se abre un abismo, un vacío, que nos muestra la crudeza del mundo precarizado, sin colchón que nos respalde o con el mismo de siempre: la familia tradicional que viene a tapar agujeros, si es que puede, una y otra vez". Gil, Silvia. 2012.

${ }^{7}$ Cita extraída de la tesis de Ana Palomo, La Maternidad en la creación plástica femenina. El caso de Ana Álvarez-Errecalde.
} 


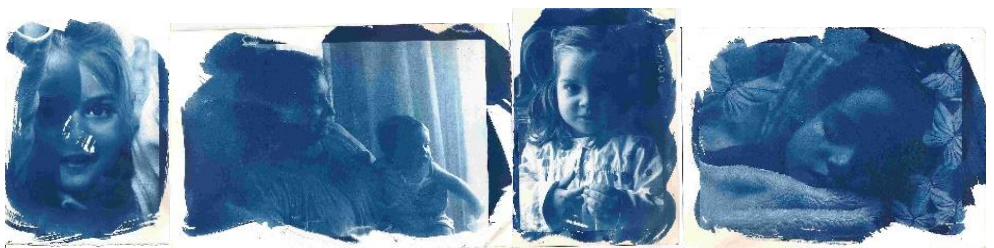

2013, Amor en azul, pintura y fotografía, cianotipias y amor en azul
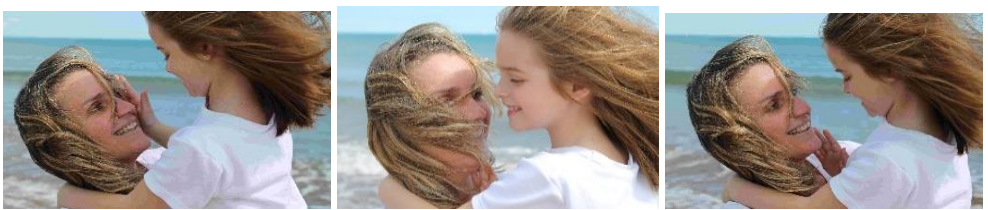

https://www.youtube.com/watch?v=0PXNqayGZc4 2014, Eva y Carola, video y fotografía
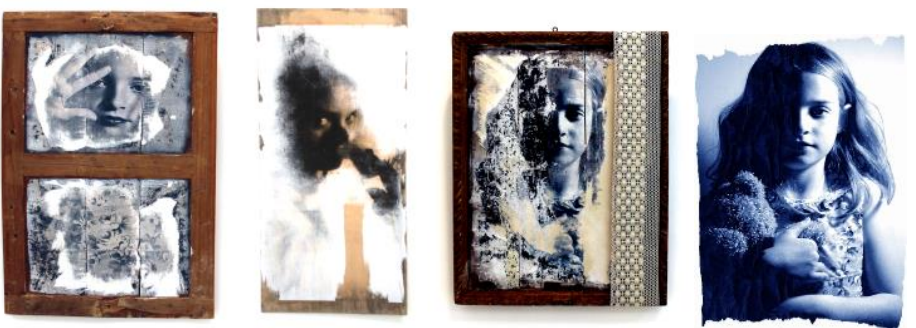

2015, Ventanas adentro, ensamblaje, pintura y fotografía
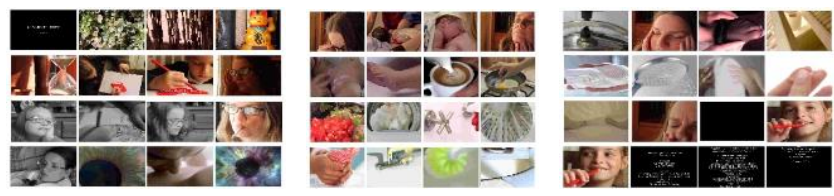

https://www.youtube.com/watch?v=ILIJ8ad_jdo\&t=163s 2015, Amor doméstico video y fotografía

Tras todos estos proyectos expuestos con anterioridad y coincidiendo con mis reflexiones y teorizaciones sobre el arte segregado de la vida y el arte del día a día, comencé a focalizar mi atención en los tiempos que estaba dedicando a los cuidados lo que ocasionó que surgiera la pregunta de si, efectivamente, podrían encontrarse manifestaciones artísticas en estos cuidados.

\section{ANTECEDENTES Y REFERENTES PARA LA INVESTIGACIÓN}

A finales del 2015, cuando comencé a trabajar en la investigación, me encontré con serias dificultades para desarrollar estado del arte del tema de la misma, puesto que no encontraba bibliografía suficiente para estructurar mi marco teórico. Pude aproximarme al tema basándome en las referencias que me proporcionó mi tutora de tesis y, posteriormente, mucha de la información sobre producción artística y reproducción, con la que estoy trabajando actualmente, la he conseguido conectándome con artistas por redes sociales. No fue hasta enero de este 2018 cuando encontré una tesis dedicada a la maternidad y el arte plástico La Maternidad en la creación plástica femenina. El caso de Ana Álvarez-Errecalde. Un estudio narrativo a propósito de la elaboración de un discurso expositivo y su materialización, la tesis del 2015, realizada por Ana María Palomo Chinarro, que me confirmó el problema al que me enfrenté desde el principio, la falta de bibliografía ${ }^{8}$ y me proporcionó mucha información sobre los referentes que se mencionan más adelante.

\footnotetext{
8 "Al iniciar la investigación sobre la maternidad en el arte contemporáneo sabía que me adentraba en un terreno inexplorado porque conocía de antemano la escasa bibliografía existente y porque presumía los vacíos teóricos que paulatinamente iría descubriendo. Si bien el camino del arte de
} 
Siguiendo una línea de tiempo desde los años sesenta en el Arte contemporáneo hasta el día de hoy a continuación detallo una serie de artistas que han aportado o aportan actualmente narrativa escrita, plástica, visual y vivencial al tema de la maternidad.

Artistas como Marina Abramović y Tracey Emin se han manifestado públicamente sobre el tema de la maternidad y otras como Aleydis Rispa, Ana Álvarez-Errecalde, Ana Casas Broda, Art al Quadrat, Elinor Carucci, Alice Neel, Alicia Leal, Ana Cardim, Ana Mendieta, Ana Sabiá, Àngels Ribé, Anna Jonsson, Annie Leibovitz, Barbara Hepworth, Barbara Kruger, Bea Sánchez, Betye Saar, Carmen Calvo, Carmen Montoro, Carmen Sigler, Carolee Schneemann, Cindy Sherman, Cori Mercadé,Courtney Kessel,Darcy Padilla,Diane Arbus, Eulàlia Valldosera, Eva Hesse, Graciela Iturbide, Hackney Flashers Collective, Heather Gray, Jacqueline Tarry y Bradley McCallum, Janine Antoni, Jenny Holzer, Jill Miller, Judy Chicago, Leila Amat, Lenka Clyton, Louise Bourgeois, Margaret Harrison, Mari Chordà, María Llopis Navarro, Maris Bustamante y Mónica Mayer en Polvo de Gallina negra, Marisa González, Marlene Dumas, Marta María Pérez Bravo, Martha Rosler, Mary Kelly, Mayte Carrasco, Monica Sjöö, Natalia Iguiñiz, Nicola Constantino, Niki de Saint Phalle, Regina José Galindo, Renee Cox,Rineke Dijkstra, Sally Man, Susan Hiller, Susan Sontag, Ulrike Rosenbach, Verónica Ruth Frías, Yurie Nagashima entre otras trabajan actualmente o han trabajado en él.

De todas las artistas mencionadas con anterioridad las dos que detallo a continuación han llamado particularmente mi atención desde el principio de la elaboración de la tesis.

\section{Mary Kelly, 1941}

En la época en la que la artista estadounidense Mary Kelly trabajó, muchas artistas feministas consideraban la maternidad incompatible con su trabajo creativo y profesional.

En Post-Partum document, realizado entre los años 1973 y 1979 durante seis años, explora la relación madre e hijo y nos la presenta con una obra conceptual influenciada por el feminismo y el psicoanálisis. La artista exploró la contradicción que se le generaba entre el desempeño de su labor artística y su labor como madre, entre sus labores creativas y sus labores reproductivas, estableciendo en su obra una diferencia entre su experiencia y el análisis de la misma.

La obra consta de seis series y contiene más de un centenar de documentos, como ropa usada de su hijo, notas de diario, gráficos y otros objetos tales como pañales sucios, grabaciones de balbuceos, regalos, huellas, moldes y un amplio registro de memoria de la relación entre madre e hijo.

Mary Kelly grabó una serie de conversaciones con su hijo, reflexionó sobre ellas y luego le permitió garabatear una serie de documentos, con ello realizó una serie compuesta de trece obras de lápiz, ceras, tiza y diagramas impresos sobre papel.

Este estudio nos permite visualizar la maternidad desde un punto de vista que se sale del instintivo para introducirnos en el universo social y cultural de la misma en donde se yuxtaponen la artista profesional y la madre, la esfera pública y la privada, prescindiendo de su imagen, de su cuerpo en relación con la maternidad, enfatizando la labor de cuidado de su hijo.

Su objetivo era el de construir una feminidad sin objetualizarla, reflejando la posición social de las madres como primeras cuidadoras, demostrando que el papel de madre podría ser un catalizador para la creación. Un trabajo que cristaliza la maternidad como un proceso psicológico y social además de biológico y emocional.

\section{Ana Álvarez-Errecalde, 1973}

La artista argentina manifiesta haber descubierto un poder catártico en el arte, trabajando desde su historia personal utilizando la fotografía el vídeo y la instalación.

Muy activa en redes nos habla sobre su trabajo artístico yuxtaponiéndolo con su día a día, aporta la interpretación que ella hace de su realidad y está pendiente de lo que sus seguidores le comentan, Álvarez-Errecalde entrama su entorno vital y su capacidad artística e interactúa en la red, lo que hace que se genere conocimiento inmediato, cambiando el imaginario colectivo de maternidad y cuidados desde un enfoque artístico sobre el tema, aunque la artista trabaja en el tema de la maternidad y crianza vinculadas al cuerpo femenino, gestación, parto y lactancia, núcleo universal de la continuación de la vida humana, lo que más me llama la atención de su trabajo precisamente es el entramado de vida y arte en el que se desarrolla y que comparte en redes con la etiqueta \#vidayarte.

género y del arte feminista, que eran las referencias que intuía más próximas, ya estaba demasiado trillado, la concreción del tema que me proponía estudiar se me presentaba como un oasis en un desierto" Ana María Palomo Chinarro. 
Mary Kelly y su obra analítica y conceptual y Ana Álvarez-Errecalde, quien utilizando su cuerpo, se retrata con su hija en brazos justo después del parto y que, con su trabajo y presencia activa en redes, apunta el camino en el que se yuxtaponen arte y vida en un mundo de comunicación global, son dos referentes fundamentales la investigación que llevo a cabo.

\section{EN BUSCA DE LA DIMENSIÓN COMÚN, NUESTROS CASOS}

El estudio de casos correspondiente a la presente investigación ha consistido en la recopilación de cincuenta encuestas, realizadas a mujeres que, además de artistas, son madres. La encuesta consta de treinta y cuatro preguntas algunas de tipo cuantitativo y otras de carácter cualitativo. A continuación se exponen algunos de los datos obtenidos.

Los años de nacimiento de las colaboradoras se encuentran entre 1940 y 1980 . El $84 \%$ de las encuestadas son de nacionalidad española y $6 \%$ son de distintas nacionalidades latinoamericanas.

El $92 \%$ del total son poseedoras de estudios universitarios o equivalentes, el resto de enseñanza profesional. Un $18 \%$ de ellas son pintoras, un $14 \%$ fotógrafas, otro $14 \%$ se definen como artistas multidisciplinares, un $6 \%$ se dedican a la docencia, un $5 \%$ a la escultura, y el porcentaje que suma las multidisciplinares más el $29 \%$ se reparte en artistas dedicadas al dibujo, grabado, ilustración, diseño, audiovisual, performance, instalación, arquitectura, restauración, dirección artística, comisariado, gestión cultural, e investigación.

Es resaltable el hecho para la cuestión que ocupa a la investigación, un $96 \%$ manifieste que la maternidad le ha cambiado la vida, indicando en un $88 \%$ el cambio de prioridades vitales tras la misma. Importante también resaltar que para el $92 \%$ de las encuestadas manifiesta como positiva la experiencia vital de la maternidad, y que, de ese $92 \%$ que la definen como una experiencia vital positiva, el $62 \%$ lo hagan de una forma rotunda y el $30 \%$ le den un carácter ambivalente a la respuesta, indicando que es una experiencia definitivamente positiva pero, a la vez, explican sus dificultades, un $6 \%$ indica serias dudas de que la maternidad sea un hecho positivo en la vida de una mujer.

Respecto a los cuidados que realiza el padre, el $50 \%$ de las madres han indicado que el padre se ha implicado igual que ellas, un $4 \%$ que lo ha hecho en menor medida que ellas, el 30\% refiere que el padre se ha implicado poco en el cuidado de los hijos, el $10 \%$ que no se ha implicado nada, el $4 \%$ indica que el padre se ha hecho cargo de los cuidados en mayor medida que ellas.

El $80 \%$ de las encuestadas manifiesta que ha delegado el cuidado de sus hijos a otras personas, externas a la pareja en el caso de unidades familiares biparentales, el $22 \%$ indica que lo ha hecho por necesidad, el $10 \%$ indica que lo ha hecho por considerarlo mejor para ella y el $34 \%$ considera que ha delegado por ambas razones, es decir, tanto por necesidad económica como por considerarlo que era beneficioso para ellas. Del $80 \%$ que ha delegado el cuidado, el $20 \%$ ha delegado en su madre (la abuela), el $26 \%$ en establecimientos públicos o privados y el $16 \%$ en una empleada de hogar entre las que el $4 \%$ son inmigrantes. El $38 \%$ restante se reparte entre sus padres (el abuelo) con un $8 \%$, familiares de segundo grado, amigas y amigos, y redes de madres.

Entrando ya en el tema de cambios artísticos tras la maternidad, un $74 \%$ de las encuestadas contestan que su obra artística ha sufrido cambios tras la maternidad, con un $66 \%$ que refiere una baja a nivel productivo. En cuanto a cambios a nivel matérico se manifiestan un $54 \%$. Me ha parecido interesante que en este punto, un $38 \%$ han referido a que han tenido que cambiar de matérico a virtual, es decir han pasado de obra plástica a fotografía y vídeo, un $19 \%$ han cambiado a soportes más pequeños, un $10 \%$ refieren a que utilizan técnicas menos peligrosas o agresivas, otro $10 \%$ refiere a que utiliza acrílico dado que seca antes, el porcentaje restante se dividen entre las que indican que han preferido hacer barro porque se podía modelar en casa, se han dedicado a la ilustración, la realización de cerámica y textil, y el uso de objetos cotidianos para ejecutar obra.

A la pregunta de si su obra había sufrido cambios a nivel discursivo tras la maternidad, un $56 \%$ refiere que sí que ha habido cambios, refieren cambio de narrativa, obra más delicada e intimista, y temas como la ecología, cotidianeidad, arte y vida, derechos humanos, educación, proyectos vinculados a los niños, lo doméstico, el género, política, cuidados, emociones, maternidad, familia y los sentimientos.

En cuanto a las preguntas de yuxtaposición de arte y cuidados, concretamente la de si alguna labor de cuidado de los hijos podría considerarse artística, un $56 \%$ manifiesta una respuesta positiva, como ejemplo la respuesta de Rojo 5 que me indica que entiende su vida como una unidad y no diferencia actitudes según labores, que no hay diferencia entre su labor artística, al menos de forma consciente y la de cuidado de sus hijos y que las labores de cuidado de sus hijos son actividades artísticas. Importante también resaltar que, a la pregunta sobre si alguna labor de crianza o cuidados les ha parecido creativa, un $82 \%$ responde afirmativamente entre las que un $28 \%$ relaciona la creatividad con la educación de sus hijos. 


\section{CONCLUSIÓN, MI TRABAJO ARTÍSTICO EN LA ACTUALIDAD}

Lo que la infancia vive determina el futuro, y el cuidado de la infancia necesita mucho tiempo de dedicación, "tiempo que tiene un alto componente relacional" (Legarretalza 2009) por tanto, necesario es pensar en un modelo de futuro en el que la reproducción humana se lleve a cabo de una manera reflexiva ${ }^{9}$.

Es importante reflexionar sobre la labor que ejercemos como madres y padres, junto con nuestros hijos, puesto que es un proceso de retroalimentación. Encontrar en nuestro modo de hacer aquello ayude a que se lleve a cabo un desarrollo vital saludable, desde una perspectiva artística, de la infancia, cuidando y respetando su materia y sin apenas intervenir en sus decisiones lo que les permitiría potenciarse y encaminarse en su propia dirección vital.

La sociedad necesita organizarse teniendo en cuenta las necesidades para el cuidado y la continuación de la vida, los hombres han de implicarse en el cambio, por lo tanto es necesario que reflexionemos y elaboremos discursos con la finalidad de crear un nuevo organismo social más justo para la infancia.

Podríamos observar casa, la ciudad, los no lugares, el mundo como "talleres" en los que desarrollamos nuestra labor como madres y padres.

Parece imprescindible es que los temas de reproducción humana y social sean investigados e impartidos a nivel académico en los ámbitos de las Ciencias Sociales, Psicología, Filosofía y Arte para complementar y equilibrar de forma transversal los conocimientos sobre reproducción humana que se están generando a nivel técnico desde ámbitos como de la biotecnología o la ingeniería biomédica ${ }^{10}$.

En mi caso particular, estoy haciendo el ejercicio de pensar en mi casa como taller y reflexionar y elaborar un discurso visual sobre un trabajo en el que se yuxtapongan los útiles, herramientas, utillajes, virutas, materia, colores, formas y objetos que aparecen en mis talleres, no porque sea el único lugar en el que desarrollo los cuidados de mi hija, como explico arriba, pero necesito focalizarme en algo para empezar a trabajar en el tema, inspirada en lo que manifestaba Judy Chicago acerca de la necesidad de experimentar hechos reales de la vida, sentimientos e inquietudes de las mujeres.

\section{DIFUSIÓN}

El tema de la maternidad es asunto cuyas narrativas se expanden a gran velocidad con el uso de las tecnologías sociales ${ }^{11}$ y sus recursos para la comunicación visual y plástica han cambiado radicalmente con el uso de las nuevas tecnologías y las redes sociales desde los años 60 hasta el presente ${ }^{12}$.

Los cambios estéticos narrativos, sobre un tema que hasta hace poco tiempo ha sido poco valorado y explorado, y el conocimiento que generan se expanden a gran velocidad.

\footnotetext{
9 “Fenómenos como el crecimiento de Internet, de los sistemas financieros, de la economía afectada por el envejecimiento y de la sociedad ecológica demuestran de forma evidente, que es necesario meditar sobre estas cuestiones y responder a ellas al mismo tiempo en sus dimensiones de inclusión e innovación. Los objetivos que Europa pretende alcanzar mediante este reto están clasificados en función del tipo de Sociedad al que van dirigidos: sociedades inclusivas; sociedades innovadoras y sociedades reflexivas" (Europa Horizonte2020).

10 "Henri Atlan, biólogo y especialista en bioética, señala una nueva y formidable frontera: aquella que verá a la especie humana reproducirse fuera del cuerpo de las mujeres." "Niños de Máquina”. EL PAís. 2017. http://elpais.com/diario/2005/06/12/eps/1118557609_850215.html?id_externo_rsoc=FB_CC.

11 "Se llama Tecnología Social a la aplicación del conocimiento científico no a la materia inerte, sino a los sistemas sociales; este concepto es sinónimo de Tecnología Blanda, es decir, el conjunto de técnicas y métodos aplicados al desarrollo de las relaciones e interacciones humanas" (Carrascosa 2016, nota número 5).

12 "Un nuevo sistema de comunicación, que cada vez habla más un lenguaje digital universal, está integrando globalmente la producción y distribución de palabras, sonidos e imágenes de nuestra cultura y acomodándolas a los gustos de las identidades y temperamentos de los individuos. Las redes informáticas interactivas crecen de modo exponencial, creando nuevas formas y canales de comunicación, y dando forma a la vida a la vez que ésta les da forma a ellas" (Castells 2005).
} 
Benedito Pérez, Carmen

Producción y reproducción, aproximación a los cuidados desde unas perspectivas y focalización artísticas

IV Congreso INTERNACIONAL DE INVESTIGACIÓN EN ARTES VISUALES ANIAV 2019

IMAGEN [N] VISIBLE]

http://dx.doi.org/10.4995/ANIAV.2019.8946

\section{FUENTES REFERENCIALES}

Butler, J. (2002). Los cuerpos que importan. En: Cuerpos que importan: Sobre los limites materiales y discursivos del sexo (p. 53-94). Traducción de Alcira Bixio. Buenos Aires-Barcelona: Paidós. https://doi.org/10.4067/S0718-71812012000200027

Carrascosa Vacas, A. (2016). La construcción del organismo social como una obra de arte (Repeating Beuys). AusArt, 4 (2), 23-34. https://doi.org/10.1387/ausart.17237

Castells Oliván, M. (2002). La era de la información: Economía, sociedad y cultura. Vol. 1: La sociedad red. Versión castellana de Carmen Martínez Gimeno y Jesús Alborés. México DF: Siglo XII.

Castells Oliván, M. (2002). La era de la información: Economía, sociedad y cultura. Vol. 2: El poder de la identidad. Versión castellana de Carmen Martínez Gimeno y Jesús Alborés. México DF: Siglo.

Federici, S. (2013). Revolución en punto cero: Trabajo doméstico, reproducción y luchas feministas. Madrid: Traficantes de Sueños.

Gil, S. (2012). Nuevos Feminismos: Sentidos Comunes En La Dispersión. Una Historia de Trayectorias Y Rupturas En El Estado Español. Hispanic Review, 80. https://doi.org/10.1353/hir.2012.0042

Fernández Pujana, I. (2014). Feminismo y maternidad ¿una relación incómoda?: Conciencia y estrategias emocionales de mujeres feministas en sus experiencias de maternidad. Vitoria-Gasteiz: Emakunde.

http://www.emakunde.euskadi.eus/contenidos/informacion/certamen publicaciones/es def/adjuntos/2013.feminismo.maternidad.r elacion.incomoda.pdf

Legarretalza Iza, M. (2009). El tiempo donado en el ámbito doméstico: Reflexiones para el análisis del trabajo doméstico y los cuidados. Cuadernos de Relaciones Laborales, 26 (2), 45-69. https://doi.org/10.5209/CRLA.33400

Monleón Pradas, M. (2010). Hacia una visibilización de la crisis de los cuidados: Arte social frente a nueva esclavitud poscolonial. Arte y Políticas de Identidad, 2, 25-44. http://revistas.um.es/api/article/download/117261/110911

Palomo Chinarro, A. M. (2015). La Maternidad en la creación plástica femenina. el caso de Ana Álvarez-Errecalde. un estudio narrativo a propósito de la elaboración de un discurso expositivo y su materialización." TDX (Tesis Doctorals En Xarxa). Universitat de Vic. http://www.tdx.cat/handle/10803/313229

Precarias a La Deriva. (2004). A La Deriva: Por Los Circuitos de La Precariedad Femenina. Madrid: Traficantes de Sueños. https://doi.org/10.1017/СВ09781107415324.004

Rich, A. (1996). Nacemos de mujer: la maternidad como experiencia e institución. Postfacio a la edición española de Mercedes Bengoechea. Madrid: Cátedra.

\section{WEBS VISITADAS}

Alonso, M. (2015). Arte, feminismo y maternidad. Arte y Cultura Visual. http://www.m-arteyculturavisual.com/2015/03/19/artefeminismo-y-maternidad/

Álvarez-Errecalde, A. (2005). “El nacimiento de mi hija”. Alvarezerrecalde.com, web personal de la artista. http://alvarezerrecalde.com/portfolio/el-nacimiento-de-mi-hija/?nacimiento?de?mi?hija/

Amat, L. (2018). Nunca Es La Hora Del Sueño - Leilaamat. http://leilaamat.com/nunca-es-la-hora-del-sueno

Art al Quadrat. (2019). web personal de las artistas. https://www.artalquadrat.net/work-2/

Carucci, E. (2014). Mother. Elinorcarucci.com, web personal de la artista. http://www.elinorcarucci.com/mother.php\#9

Casas Broda, A. (2013). "Kinderwunsch". Anacasasbroda.com, web personal de la artista.

http://www.anacasasbroda.com/kinderwunsch

Harrison, M. (2017). Work. Margaret-harrison.com, web personal de la artista. http://margaret-harrison.com/?gallery=work-2 
Benedito Pérez, Carmen

Producción y reproducción, aproximación a los cuidados desde unas perspectivas y focalización artísticas

IV Congreso INTERNACIONAL DE INVESTIGACIÓN EN ARTES VISUALES ANIAV 2019

IMAGEN [N] VISIBLE]

http://dx.doi.org/10.4995/ANIAV.2019.8946

Hiller, S. (1979). Ten Months. 2017. Susanhiller.org, web personal de la artista.

http://www.susanhiller.org/otherworks/ten months.html

Kelly, M. (1973-79) 2015. Post partum document. Marykellyartist.com, web personal de la artista, última modificación 5 abril. http://www.marykellyartist.com/post partum document.html

Llopis, M. (2010). El embarazo como el mega estadio sexual de los cuerpos. Mariallopis.com, web personal de la artista. https://www.mariallopis.com/portfolio/el-embarazo-como-el-mega-estadio-sexual-de-los-cuerpos/

Mann, S. (1984). Family Pictures. Sallymann.com, web personal de la artista. http://sallymann.com/selected-works/family-pictures

Pardo S, Rebeca. (2012). La maternidad en el arte: Visiones renovadas tras el Post Partum Document. En la retaguardia: Imagen, Identidad y memoria (blog), 6 junio.

https://rebecapardo.wordpress.com/2012/05/06/la?maternidad?en?el?arte?visiones?renovadas?tras?el?post?partum?document/

Sabiá, A. (2013). Madonnas contemporáneas. Anasabia.com, web personal de la artista. https://www.anasabia.com/madonnascontemporaneas-2012-13

Sanchez, B. (2016). No sé cómo puedes con todo II. Beasanchez.es, web personal de la artista.

http://www.beasanchez.es/momisinthestudio/no-se-como-puedes-con-todo-ii/ 